Pflegemindestlohn

\section{Private Pflegeunternehmen: Absage an flächendeckende Tarifverträge}

\) Der Mindestlohn in der Altenpflege ist zum 1. Januar 2018 erneut angestiegen. Im Westen liegt er jetzt bei 10,55 Euro, im Osten bei 10,05 Euro. Er betrifft vor allem Pflegehilfskräfte und Betreuer. Friedhelm Fiedler, Vizepräsident des Arbeitgeberverbandes Pflege: „Seit Einführung des Pflegemindestlohns im August 2010 haben wir regelmäßig kräftige Steigerungen erlebt. Damals lag der Pflegemindestlohn für ungelernte Kräfte bei 8,50 Euro im Westen und 7,50 Euro im Osten." Diese Steigerungen seien ein Kraftakt. Aber gutes Personal solle auch fair bezahlt werden. Allen Bestrebungen flächen- deckend allgemeinverbindliche Tarifverträge „durchdrücken“ zu wollen, erteilte Fiedler eine Absage. „Die Tarifautonomie darf nicht durch eine emotionsgetriebene Politik unterminiert werden. Tarifautonomie ist ein hohes Gut und muss geschützt werden." Der Verband fordere zudem, dass die Kommission, zum Pflegemindestlohn künftig fairer besetzt werde. Statt bisher nur einen Sitz für die privaten Unternehmen auf der Arbeitgeberbank hält Fiedler zwei Sitze für angemessen.

\section{www.arbeitgeberverband-pflege.de}

\section{Rheinland-Pfalz}

\section{Landespflegekammer mit neuer Geschäftsführung}

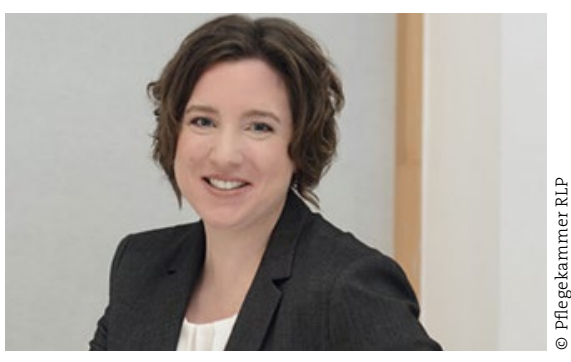

» Dr. Susanne Wagenmann hat zum 1. Dezember 2017 die Leitung der Geschäftsstelle der rheinland-pfälzischen Pflegekammer in Mainz übernommen. Wagenmann hat nach ihrem abgeschlossenen Studium der Volkswirtschaftslehre in Heidelberg an der Universität Rostock „Zur Effizienz des deutschen Gesundheitssystems" promoviert. Nach beruflichen Stationen im Wissenschaftsbereich war sie in verschiedenen Funktionen bei der Kassenärztlichen Vereinigung Rheinland-Pfalz tätig.

„Wir haben mit Frau Dr. Wagenmann eine erfahrene, motivierte und überaus kompetente Persönlichkeit für diese Aufgabe gewinnen können, die, aufgrund ihrer bisherigen beruflichen Laufbahn, gesundheitspolitisch äußerst bewandert ist und daher mit den Herausforderungen, vor denen unsere Berufsgruppe steht, vertraut ist“, kommentierte Kammerpräsident Dr. Markus Mai.

www.pflegekammer-rlp.de

\title{
Länder für gleichen Pflegemindestlohn in Ost und West
}

\section{Arbeits- und Sozialminister tagten in Potsdam}

") Neben Kinderarmut, Umgang mit Opfern terroristischer Gewalttaten, die Umsetzung des Bundesteilhabegesetzes und die Arbeitsmarktintegration geflüchteter Menschen waren die Mindestentgelte in der Pflege eines der zentralen Themen auf der 94. Konferenz der Arbeits- und Sozialminister der Länder Anfang Dezember 2017. Unter dem Vorsitz des Landes Branden- burg, das als Gastgeber fungierte, forderten die Länder einen einheitlichen Mindestlohn in Ost und West. Ihrer Auffassung nach muss die regionale Differenzierung beendet werden, da es für sie 30 Jahre nach der Wiedervereinigung keine sachliche Rechtfertigung mehr gebe. Es sei „den Pflegekräften in Ostdeutschland nicht mehr vermittelbar, dass sie weniger verdienen sollen als ihre westdeutschen Kolleginnen und Kollegen. Sowohl der Arbeits- und Zeitumfang, die hohe Verantwortung als auch die physischen und psychischen Belastungen am Arbeitsplatz sind unabhängig vom Arbeitsort identisch“, heißt es in der Begründung zum Beschluss.

www.masgf.brandenburg.de 